02

\title{
Образование свободных электронов при столкновении Хе с альфа-частицами
}

\author{
(C) А.А. Басалаев, М.Н. Панов, О.В. Смирнов \\ Физико-технический институт им. А.Ф. Иофффе РАН, \\ 194021 Санкт-Петербург, Россия \\ e-mail: m.panov@mail.ioffe.ru
}

Поступило в Редакцию 18 марта 2020 г.

В окончательной редакции 8 июня 2020 г.

Принято к публикации 8 июня 2020 г.

Измерены сечения элементарных процессов при столкновении налетающих ионов ${ }^{3} \mathrm{He}^{2+}$ с кинетическими энергиями в диапазоне $1-100 \mathrm{keV}$ с атомами Хе, сопровождающиеся образованием свободных электронов и ионов в различных конечных зарядовых состояниях: $\mathrm{Xe}^{n+}(n=1-6)$ и $\mathrm{He}^{(2-m)+}(m=0-2)$. Выделены процессы ионизации, когда налетающая альфа-частица не меняет зарядового состояния, и процессы захвата одного или двух электронов, ведущие к образованию однозарядного иона или атома гелия с дополнительным удалением электронов из атома ксенона в сплошной спектр. Определены сечения образования свободных электронов в каждом из этих процессов. Определен вклад в образование свободных электронов многоэлектронных процессов. Показано, что при изменении энергии столкновения ионов ${ }^{3} \mathrm{He}^{2+}$ от 1 до $100 \mathrm{keV}$ (скорости налетающего иона от 0.12 до 1.2 a.u.) принципиально меняется механизм удаления электронов из атома ксенона. При малых скоростях сближения частиц происходит образование автоионизационного состояния квазимолекулы и его последующий распад. При больших скоростях ( $V>0.7$ a.u.) образование свободных электронов в основном обусловлено внезапным изменением потенциальной энергии электронов при сближении быстрой альфа-частицы с ядром атома ксенона.

Ключевые слова: захват с ионизацией, многоэлектронные процессы, свободные электроны, квазимолекулярный механизм.

DOI: $10.21883 / \mathrm{JTF} .2020 .12 .50116 .92-20$

\section{Введение}

При столкновении ионов с атомами одним из наиболее изучаемых процессов является процесс образования свободных электронов. С точки зрения теоретического рассмотрения процесса ионизации, столкновение многоэлектронного атома с бесструктурной частицей протоном или альфа-частицей - является наиболее простым. В цикле статей [1-3] показано, что даже для случая столкновений многоэлектронных атомов с протонами экспериментальные данные, детальные по каналам процессов, довольно бедны, особенно в низкоэнергетической области, что затрудняет оценку теоретических вычислений. Имеющиеся в настоящее время теоретические подходы для расчета сечений ионизации атомов достаточно хорошо развиты в одноэлектронном приближении в области высоких энергий налетающих частиц, т. е. когда скорость налетающего иона $\left(V_{p}\right)$ больше, чем начальная скорость электрона $\left(V_{e l}\right)$, участвующего в процессе эмиссии. При столкновении с многоэлектронным атомом налетающих альфа-частиц с кинетическими энергиями в сотни $\mathrm{keV}$ удовлетворительное согласие с экспериментальными данными о величинах сечений и о зарядовом составе образующихся из атомов мишени медленных ионов дает механизм взаимодействия, рассматривающий быстрое изменение потенциала при сближении сталкивающихся частиц, в котором находят- ся электроны атома-мишени, с последующей эволюцией электронов возбужденного атома $[3,4]$.

В ряде работ [5-10] использовалась модель, в которой столкновение рассматривалось как взаимодействие протона или альфа-частицы с множеством слабо связанных электронов. Вероятность ионизации получалась путем использования плазменного приближения с учетом энергии связи для каждой оболочки. При описании многоэлектронной ионизации учитывались Оже-процессы, сопровождающие удаление внутреннего электрона $[5,8,11,12]$. В работах [7-9] для вычисления многократной ионизации тяжелых атомов, бомбардируемых ядрами элементов, использовалось уравнение статистической физики для описания прохождения заряженной частицы в облаке электронов неоднородной плотности, отражающей различные электронные оболочки атомамишени. Получено хорошее согласие с экспериментом $[3,4]$ для сечения ионизации $\left(\mathrm{H}^{+}-\mathrm{Xe}\right)$ с образованием от одного до четырех электронов при скоростях сближения частиц свыше $1 \mathrm{MeV} / \mathrm{a} . \mathrm{m} . \mathrm{u}$.

В этой области высоких скоростей сближения частиц имеется так же ряд квантово-механических методов расчета. Метод искаженных волн [8,13-15] дает хорошие результаты для процесса однократной ионизации атомов $\mathrm{Ar}$ и $\mathrm{Ne}$ при высоких энергиях столкновений с протонами и многозарядными ионами ряда легких элементов. При энергиях столкновения $\sim 1 \mathrm{MeV} /$ a.m.u. применение 
модели независимых частиц $[6,8,9,15,16]$ и вычисление сечения процессов многократной ионизации, в которых важную роль играет ионизация внутренних оболочек, сопровождаемая последующим Оже-распадом образующегося возбужденного иона.

Однако в области энергий столкновений в десятки keV/a.m.u. и ниже нет адекватных методов расчета сечений процессов образования нескольких свободных электронов, поэтому имеется потребность в измерении детальных характеристик процесса ионизации, в том числе зависимости сечений элементарных процессов, ведущих к образованию свободных электронов, от энергии сталкивающихся частиц, а также в измерении дифференциальных сечений рассеяния для процессов с различной степенью ионизации атома-мишени. Измерение зависимости сечений элементарных процессов изменения зарядового состояния от энергии столкновений позволяет выделить ту область энергий столкновений, в которой механизм процессов образования свободных электронов, основанный на адиабатических электронных переходах в квазимолекуле, сменяется взаимодействием энергичной альфа-частицы с квазинезависимыми электронами многоэлектронного атома мишени. В случае адиабатического сближения частиц имеется возможность рассмотрения процесса как изменения электронных уровней квазимолекулы и расчета вероятностей электронных переходов между ними. Процесс образования свободных электронов при этом может происходить как за счет кинетической энергии налетающей частицы, так и за счет потенциальной энергии квазимолекулярной системы со свободным глубоким электронным уровнем, образующейся при сближении многоэлектронного атома с многозарядным ионом. При столкновении иона с многоэлектронным атомом его ионизация происходит в основном в многоэлектронных процессах и может сопровождаться захватом электронов налетающим ионом $[17,18]$, т. е. при столкновении с $\alpha$-частицей - захватом одного или двух электронов с дополнительной ионизацией атома.

\section{1. Методика эксперимента}

При столкновении иона $\mathrm{He}^{2+} \mathrm{c}$ атомом Хе процессы образования свободных электронов могут быть разделены на три группы в зависимости от количества электронов $(m)$, захваченных налетающим ионом:

$$
\mathrm{He}^{2+}+\mathrm{Xe} \rightarrow\left\{\begin{array}{l}
\mathrm{He}^{2+}+\mathrm{Xe}^{n+}+n e^{-} \\
(1) \text { ионизация }\{220 n\}, \\
\mathrm{He}^{+}+\mathrm{Xe}^{n+}+(n-1) e^{-} \\
(2) \text { захват с ионизацией }\{210 n\}, \\
\mathrm{He}^{0}+\mathrm{Xe}^{n+}+(n-2) e^{-} \\
(3) \text { двойной захват } \\
\text { с ионизацией }\{200 n\} .
\end{array}\right.
$$

При обозначении процессов будут использованы четыре числа, соответствующие начальным и конечным зарядовым состояниям взаимодействующих частиц $\{2(2-m) 0 n\}$, сечения соответствующих процессов будут обозначаться как $\sigma_{0 n}^{22-m}$. При измерениях использовался изотоп ${ }^{3} \mathrm{He}$ для того, чтобы избежать попадания в состав первичного пучка $\mathrm{He}^{2+}$ примеси ионов $\mathrm{H}_{2}^{+}$, совпадающих по отношению заряда к массе с ионами ${ }^{4} \mathrm{He}^{2+}$.

Использованная для измерения абсолютных величин сечений экспериментальная методика подробно описана в наших работах [19-22] и состоит из нескольких этапов. Первоначально производилось измерение суммарной абсолютной величины сечения образования положительного заряда всех медленных ионов потенциальным методом, т.е. измерялся ток положительных ионов ксенона, образованных при прохождении пучка ионов $\mathrm{He}^{2+}$ известной интенсивности через однородную газовую мишень атомов Хе на фиксированном участке длины камеры столкновений. Сбор вторичных ионов осуществлялся с помощью однородного поперечного электрического поля. Низкая плотность газовой мишени гарантировала однократность столкновения быстрой частицы с атомами ксенона. Плотность газовой мишени измерялась абсолютно манометром Мак-Леода.

На втором этапе медленные положительные ионы Хе, образованные в газовой мишени, ускорялись поперечным электрическим полем, фокусировались на выходную щель магнитного анализатора и разделялись по заряду. С целью увеличения чувствительности установки при измерении интенсивности потока вторичных ионов высокой зарядности в качестве газа мишени использовался изотоп ксенона ${ }^{129} \mathrm{Xе.} \mathrm{Полный} \mathrm{сбор} \mathrm{вторичных} \mathrm{ионов,}$ осуществляемый фокусирующими электронными линзами, контролировался снятием вольт-амперной характеристики электростатической фокусирующей системы. В результате этого определялись сечениях образования медленных ионов $\mathrm{Xe}^{n+}$ определенного заряда Измерения интенсивности пучка быстрых ионов $\mathrm{He}^{2+}$ и вторичных ионов ксенона проводились в токовом режиме.

Каждое сечение образования медленного иона $\mathrm{Xe}^{n+}$ определенного заряда являлось суммой трех элементарных сечений по всем конечным зарядам быстрых гелиевых частиц. Для разделения этого сечения на сечения элементарных процессов медленные ионы определенного заряда, выделенные магнитным анализатором, направлялись в детектор счета отдельных частиц. Пучок налетающих ионов, прошедших газовую мишень, направлялся в 30 градусный цилиндрический электростатический анализатор и после разделения по заряду также регистрировался в счетном режиме. Импульсы с детекторов быстрых и медленных ионов поступали в схему совпадений. В результате измерялся относительный вклад процессов ионизации, захвата одного электрона и захвата двух электронов в сечение образования медленного иона ксенона определенного заряда. Эффективность регистрации ионов $\mathrm{He}^{2+}$ и $\mathrm{He}^{+}$детектором отдельных частиц контролировалась путем сравнения 
интенсивности счета и величины тока, измеряемого либо в токовом режиме, либо в режиме накопления заряда.

Погрешность в абсолютных величинах сечений определялась главным образом погрешностью измерений плотности частиц газа мишени и составляла 10\%. Дополнительно возникали погрешности из-за статистики истинных и случайных совпадений импульсов детекторов, которая сильно зависит как от вида элементарного процесса, так и от величины его сечения. Для процессов (2) и (3) статистическая погрешность не более $5 \%$ для сечений порядка $10^{-17} \mathrm{~cm}^{2}$. Минимальная измеряемая величина сечения процессов 2 и 3 составляет $\sim 10^{-19} \mathrm{~cm}^{2}$, а для процесса ионизации $(1)-10^{-18} \mathrm{~cm}^{2}$ ввиду большой загрузки канала быстрых частиц ионами первичного пучка, не испытавшими взаимодействий с атомами ксенона.

\section{2. Результаты измерений и их обсуждение}

Экспериментальные величины сечений элементарных процессов изменения зарядовых состояний частиц $\sigma_{0 n}^{22-m}$ при столкновении ионов ${ }^{3} \mathrm{He}^{2+}$ в диапазоне кинетических энергий $1-100 \mathrm{keV}$ с атомами Хе, представлены в табл. 1-3.

Полные сечения процессов $1-3\left(\sigma_{t o t}^{m}, m=0-2\right)$ приведены на рис. 1.

$$
\sigma_{t o t}^{m}=\sum_{n} \sigma_{0 n}^{22-m}
$$

Из приведенных данных видно, что во всем исследованном диапазоне энергий столкновений процесс захвата с ионизацией имеет самое большое сечение среди всех процессов, при осуществлении которых образуются свободные электроны. Полное сечение процесса ионизации растет с увеличением энергии столкновений и при энергии $\sim 55 \mathrm{keV}$ сравнивается по величине с полным сечение процесса захвата двух электронов с ионизацией,

Таблица 1. Сечения каналов процесса ионизации $\{220 n\}$ $(n=1-6)\left(10^{-17} \mathrm{~cm}^{2}\right)$

\begin{tabular}{r|c|c|c|c|c}
\hline$E, \mathrm{keV}$ & $\{2201\}$ & $\{2202\}$ & $\{2203\}$ & $\{2204\}$ & $\{2205\}$ \\
\hline 9.5 & 2.48 & 0.23 & 0.07 & & \\
13.9 & 4.61 & 0.30 & 0.10 & & \\
20.2 & 4.92 & 0.50 & 0.10 & 0.05 & 0.02 \\
25.3 & 5.51 & 0.66 & 0.17 & 0.07 & 0.03 \\
33.1 & 7.46 & 0.80 & 0.20 & 0.07 & 0.04 \\
38.9 & 9.53 & 0.90 & 0.28 & 0.14 & 0.05 \\
47.1 & 14.73 & 1.47 & 0.35 & 0.14 & 0.05 \\
54.0 & 21.58 & 1.78 & 0.50 & 0.17 & 0.07 \\
60.1 & 27.07 & 2.49 & 0.58 & 0.19 & 0.08 \\
69.9 & 36.39 & 3.20 & 1.08 & 0.36 & 0.18 \\
80.2 & 46.04 & 4.50 & 1.31 & 0.43 & 0.18 \\
89.8 & 57.37 & 5.98 & 1.72 & 0.55 & 0.21 \\
99.7 & 67.33 & 7.04 & 1.89 & 0.64 & 0.24
\end{tabular}

Таблица 2. Сечения каналов процесса захвата одного электрона с ионизацией $\{210 n\}(n=2-6)\left(10^{-17} \mathrm{~cm}^{2}\right)$

\begin{tabular}{r|c|r|c|c|c}
\hline$E, \mathrm{keV}$ & $\{2102\}$ & $\{2103\}$ & $\{2104\}$ & $\{2105\}$ & $\{2106\}$ \\
\hline 1.0 & 47.70 & & & & \\
3.0 & 35.00 & 6.95 & & & \\
5.9 & 28.10 & 7.70 & 0.60 & & \\
9.5 & 27.00 & 8.46 & 1.18 & 0.05 & \\
13.9 & 26.60 & 9.40 & 2.00 & 0.10 & 0.02 \\
20.2 & 27.00 & 10.84 & 3.01 & 0.34 & 0.03 \\
25.3 & 28.80 & 10.86 & 5.24 & 1.06 & 0.10 \\
33.1 & 32.10 & 11.24 & 5.98 & 2.14 & 0.25 \\
38.9 & 33.90 & 11.63 & 6.39 & 2.55 & 0.32 \\
47.1 & 38.20 & 12.27 & 6.61 & 3.07 & 0.43 \\
54.0 & 41.30 & 13.03 & 7.16 & 3.34 & 0.50 \\
60.1 & 43.80 & 13.74 & 7.31 & 3.50 & 0.57 \\
69.9 & 46.00 & 15.18 & 8.07 & 3.74 & 0.69 \\
80.2 & 46.50 & 16.55 & 8.36 & 3.95 & 0.82 \\
89.8 & 47.40 & 17.03 & 8.32 & 3.88 & 0.95 \\
99.7 & 46.50 & 17.06 & 8.50 & 3.89 & 0.98
\end{tabular}

Таблица 3. Сечения каналов процесса захвата двух электронов с ионизацией $\{200 n\}(n=3-6)\left(10^{-17} \mathrm{~cm}^{2}\right)$

\begin{tabular}{r|r|r|r|l}
\hline$E, \mathrm{keV}$ & $\{2003\}$ & $\{2004\}$ & $\{2005\}$ & $\{2006\}$ \\
\hline 1.0 & 64.50 & 4.43 & & \\
3.0 & 38.05 & 5.18 & & \\
5.9 & 25.74 & 7.00 & 0.67 & \\
9.5 & 21.91 & 9.39 & 0.99 & 0.09 \\
13.9 & 19.02 & 10.63 & 1.42 & 0.13 \\
20.2 & 15.27 & 11.73 & 2.09 & 0.24 \\
25.3 & 14.19 & 11.23 & 4.23 & 0.77 \\
33.1 & 12.87 & 11.10 & 7.71 & 1.47 \\
38.9 & 11.22 & 10.55 & 7.77 & 1.84 \\
47.1 & 11.12 & 9.71 & 7.29 & 1.97 \\
54.0 & 9.65 & 8.70 & 6.49 & 1.92 \\
60.1 & 8.71 & 8.32 & 6.21 & 1.85 \\
69.9 & 7.52 & 7.04 & 5.35 & 1.75 \\
80.2 & 6.62 & 5.99 & 4.80 & 1.70 \\
89.8 & 5.92 & 5.21 & 4.51 & 1.53 \\
99.7 & 5.36 & 4.82 & 4.23 & 1.51
\end{tabular}

а при энергии $\sim 100 \mathrm{keV}-$ с полным сечением процесса захвата одного электрона с ионизацией. На рис. 2 приведены сечения образования свободных электронов $\sigma_{e l}^{m}$ в процессах (1-3), вычисленные на основании сечений, приведенных в табл. 1-3, согласно выражению:

$$
\sigma_{e l}^{m}=\sum_{n=m+1}^{n=6}(n-m) \sigma_{0 n}^{22-m} .
$$

Как видно из приведенных данных, несмотря на рост сечения процесса ионизации во всем исследованном диапазоне энергий столкновений, большая часть свободных электронов при взаимодействии ионов $\mathrm{He}^{2+}$ с атомами Хе образуется за счет вклада многоэлектронных кана- 
лов процесса захвата одного электрона. При энергии столкновений меньше $20 \mathrm{keV}$ образование свободных электронов в равной мере происходит за счет процессов захвата одного и двух электронов с ионизацией (рис. 2).

При энергиях столкновений выше $20 \mathrm{keV}$ сечения каналов процесса ионизации быстро растут с энергией налетающего иона (табл. 1). Уже при энергии налетающей альфа-частицы $20 \mathrm{keV}$ в процессе ионизации наблюдаются каналы, ведущие к удалению из атома Хе до пяти электронов. Обнаружено, что все сечения каналов процесса ионизации $\{220 n\}$ имеют

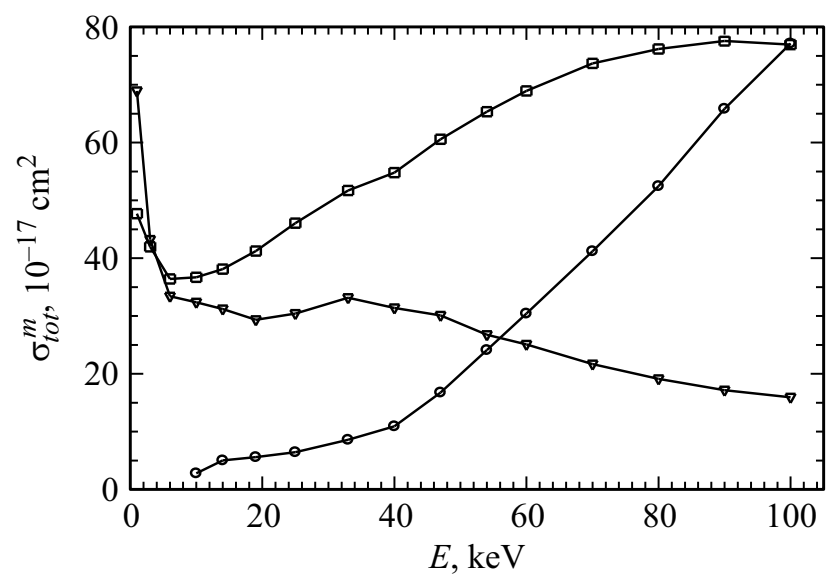

Рис. 1. Полные сечения $\left(\sigma_{t o t}^{m}\right)$ процессов ионизации, захвата одного электрона с ионизацией и захвата двух электронов с ионизацией. $\square-$ полное сечение процесса захвата одного электрона с ионизацией $\{210 n\}(n=2-6), \nabla-$ полное сечение процесса захвата двух электронов с ионизацией $\{200 n\}$ $(n=3-6)$, о - полное сечение процесса ионизации $\{220 n\}$ $(n=1-5)$.

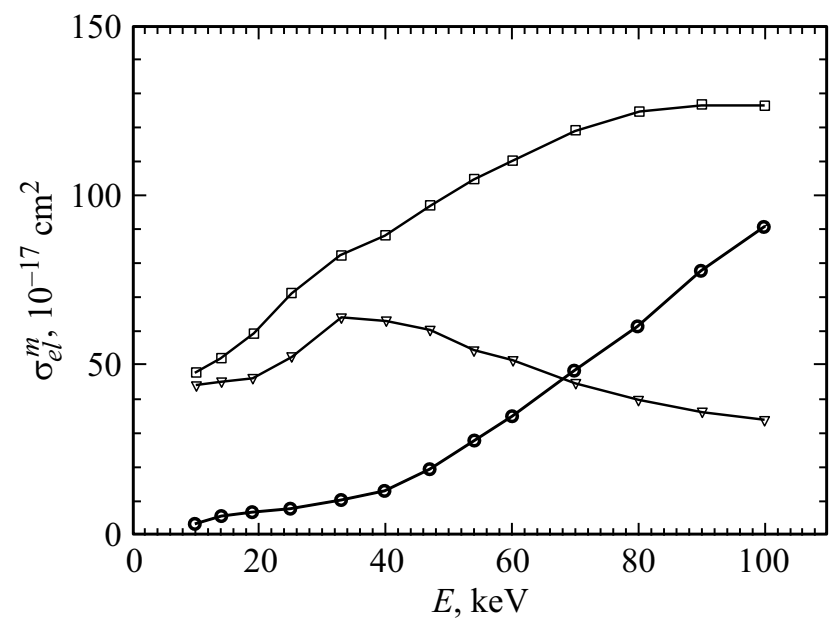

Рис. 2. Сечения образования свободных электронов в процессах ионизации, захвата одного электрона с ионизацией и захвата двух электронов с ионизацией. $\square-$ сечение образования свободных электронов $\sigma_{e l}^{1}$ в процессе $\{210 n\}$ $(n=2-5), \nabla-$ сечение образования свободных электронов $\sigma_{e l}^{2}$ в процессе $\{200 n\}(n=3-6)$, о- сечение образования свободных электронов $\sigma_{e l}^{0}$ в процессе $\{220 n\}(n=1-6)$.

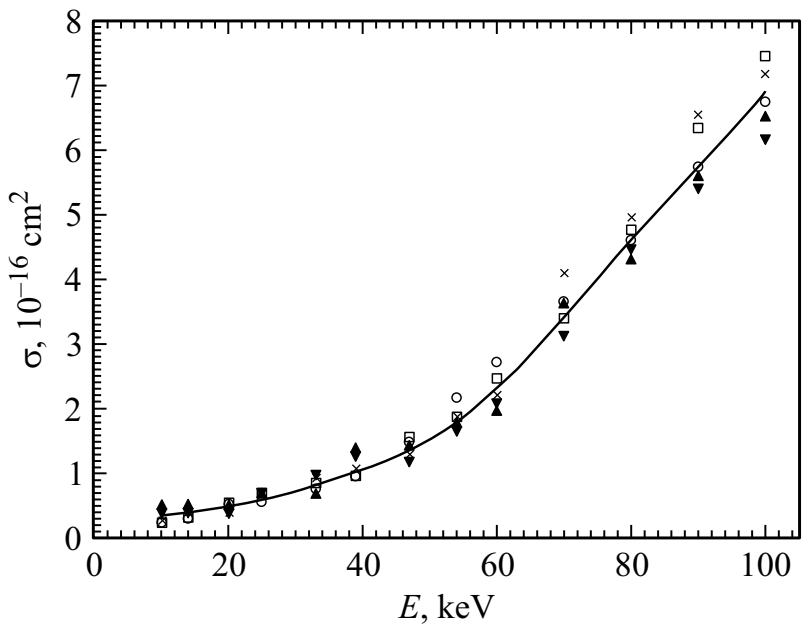

Рис. 3. Сечения каналов процесса ионизации атомов Хе ионами ${ }^{3} \mathrm{He}^{2+}$ (процессы $\left.\{220 n\}\right)$, нормированные на сечение процесса $\{2201\}$. ○- $\sigma_{01}^{22}, \square-10.6 \cdot \sigma_{02}^{22}, \times-38 \cdot \sigma_{03}^{22}, \boldsymbol{\Delta}-$ $101 \cdot \sigma_{04}^{22}, \boldsymbol{\nabla}-168 \cdot \sigma_{05}^{22}$.

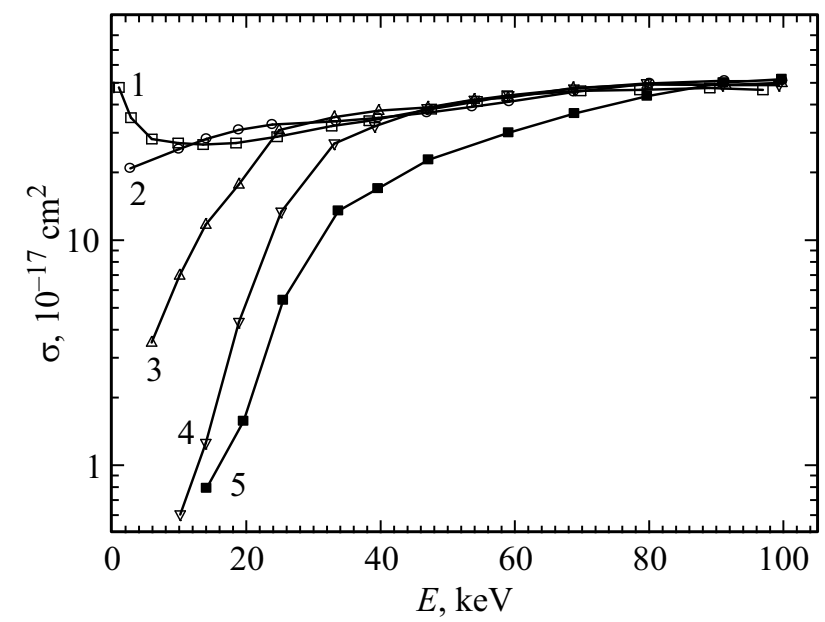

Рис. 4. Сечения каналов процесса захвата одного электрона с ионизацией атомов Хе ионами ${ }^{3} \mathrm{He}^{2+}$ (процессы $\left.\{210 n\}\right)$, нормированные на сечение процесса $\{2102\}$. (Цифры у кривых соответствуют количеству свободных электронов, образующихся в процессе). $\square-\sigma_{02}^{21}, \circ-2.78 \cdot \sigma_{03}^{21}, \boldsymbol{\Delta}-5.7 \cdot \sigma_{04}^{21}$, $\boldsymbol{\nabla}-12.2 \cdot \sigma_{05}^{21}, \boldsymbol{\square}-51 \cdot \sigma_{06}^{21}$.

близкую зависимость от энергии ионов $\mathrm{He}^{2+}$, т.е. величины сечений образования ионов ксенона любого заряда $(n=0-5)$, умноженные на постоянные коэффициенты $\left(\sigma_{01}^{22}: \sigma_{02}^{22}: \sigma_{03}^{22}: \sigma_{04}^{22}: \sigma_{05}^{22}=1: 10.6: 38: 101: 168\right)$, ложатся на универсальную кривую (рис. 3). Это объясняется тем, что все каналы процесса ионизации имеют одинаковый механизм, а именно возбуждение электронов атома в результате резкого изменения потенциала, в котором они находятся при сближении альфа-частицы и ядра атома, и последующего распада этого возбужденного состояния. При этом отсутствуют какие-либо другие виды электронных переходов, так как экспериментально выделены столкновения, в которых налетающие ионы 


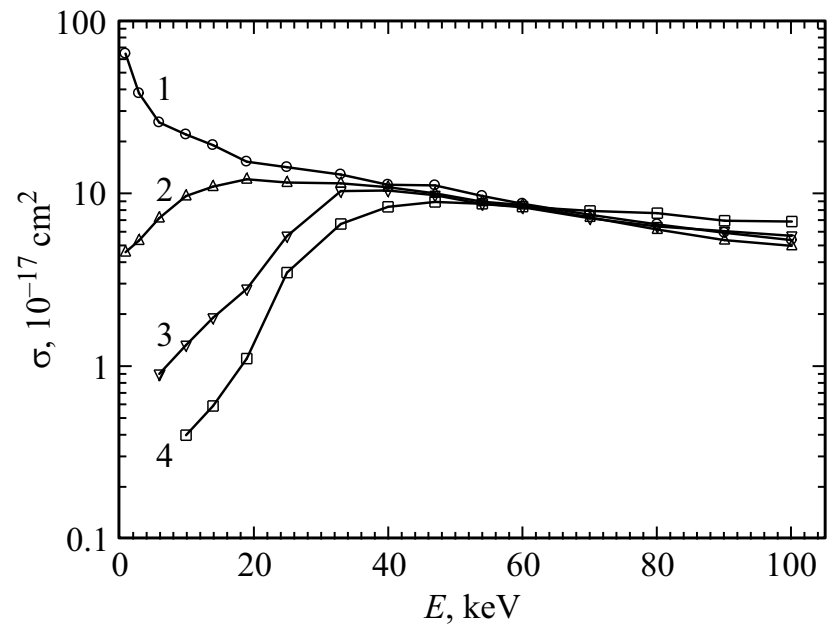

Рис. 5. Сечения каналов процесса захвата двух электронов с ионизацией атомов Хе ионами ${ }^{3} \mathrm{He}^{2+}$ (процессы $\left.\{200 n\}\right)$, нормированные на сечение процесса $\{2003\}$. (Цифры у кривых соответствуют количеству свободных электронов, образующихся в процессе). $о-\sigma_{03}^{20}, \boldsymbol{\Delta}-\sigma_{04}^{20} \cdot 1.05, \boldsymbol{\nabla}-1.4 \cdot \sigma_{05}^{20}$, $\square-4.71 \cdot \sigma_{06}^{20}$.

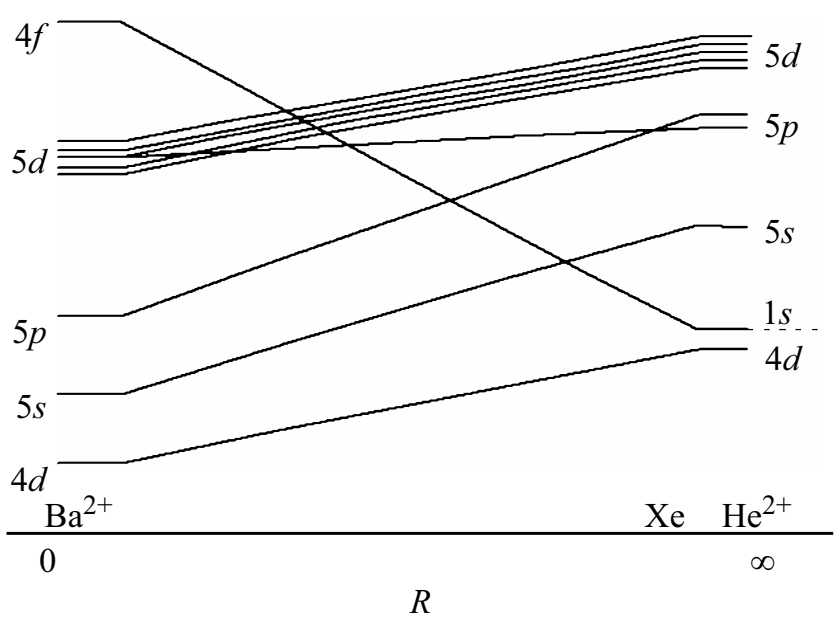

Рис. 6. Корреляционная диаграмма одноэлектронных термов квазимолекулярной системы $\{\mathrm{XeHe}\}^{2+}$.

не изменяют своего зарядового состояния, и процесс ионизации происходит только за счет возбуждения электронных оболочек. Вероятность возникновения возбужденного состояния зависит от энергии столкновения, а его последующий распад ведет к образованию свободных электронов, число которых зависит от энергии возбуждения образовавшегося молекулярного иона.

При малых скоростях сближения $(V<0.5$ a.u., $E<30 \mathrm{keV}$ ) высока вероятность захвата одного (процесс 2) или двух электронов (процесс 3). На основании величин сечений $\left(\sim 10-15 \mathrm{~cm}^{2}\right)$ этих процессов можно сделать вывод, что захват электронов в области низких энергий столкновений происходят при значительных расстояниях сближения частиц в несколько атомных единиц, т.е. затрагивает электроны лишь внешней
$5 p$-оболочки атома. В области энергий столкновений менее $50 \mathrm{keV}$ (скорости столкновения менее 0.8 a.u.) наблюдается резкое изменение зарядового состава образующихся медленных ионов ксенона в процессах, обусловленных захватом электронов. Очевидно, степень возбуждения при осуществлении процессов захвата более глубоких оболочек сильно зависит от скорости налетающих ионов. В связи с этим в отличие от процесса ионизации близкие зависимости сечений образования различного числа электронов от энергии столкновения в рассматриваемых процессах наблюдаются лишь при энергиях более $55 \mathrm{keV}$ (рис. 4,5). Для процесса захвата одного электрона и двух электронов соответственно $\sigma_{02}^{21}: \sigma_{03}^{21}: \sigma_{04}^{21}: \sigma_{05}^{21}: \sigma_{06}^{21}=1: 2.78: 5.7: 12.2: 51$ и $\sigma_{03}^{20}: \sigma_{04}^{20}: \sigma_{05}^{20}: \sigma_{06}^{20}=1: 1.05: 1.4: 4.71$.

Механизм взаимодействия иона $\mathrm{He}^{2+}$ с атомом ксенона носит при низких скоростях сближения частиц адиабатический характер и может быть качественно рассмотрен на основе анализа корреляционной диаграммы одноэлектронных термов квазимолекулярной системы $\{\mathrm{XeHe}\}^{2+}[16-18]$. При предельном сближении частиц система электронных уровней квазимолекулы $\{\mathrm{XeHe}\}^{2+}$ преобразуется в систему уровней иона $\mathrm{Ba}^{2+}$. При этом незанятая $1 s$-орбиталь гелия коррелирует с $4 f$-орбиталью образующегося объединенного иона $\mathrm{Ba}^{2+}$. $(2 p$ - и $3 d$-орбитали полностью заняты электронами Хе и расположены ниже по шкале потенциальной энергии, чем $1 s$-орбитали Не). При сближении ядер атомов $1 s-4 f$ терм квазипересекается с $5 l$ термами атома Хе. В области этих квазипересечений осуществляются электронные переходы на свободные уровни квазимолекулы. В этой области энергий столкновения с высокой вероятностью происходят процессы, связанные с захватом одного или двух электронов налетающим ионом гелия. При этом каналами образования свободных электронов могут являться процессы, обусловленные выходом диабатического терма квазимолекулы в континуум (процесс 1) [16] и автоионизации квазимолекулы как результат резонансного коррелированного двухэлектронного перехода между термами квазимолекулы и последующего распада автоионизационного состояния (процесс 2) [18]. В этой области скоростей сближения частиц наблюдается сильное изменение зарядового состава медленных ионов в процессах захвата электронов. Величины сечений захвата электронов достигают своих максимальных значений при энергиях в несколько $\mathrm{keV}$. C ростом кинетической энергии альфа-частиц растет возбуждение более глубоких оболочек и, как следствие, растет сечение ионизации. Начиная с кинетических энергий свыше $\sim 55 \mathrm{keV}$ в процессах одноэлектронного и двухэлектронного захвата наблюдается, аналогично процессу ионизации, одинаковая зависимость сечений образования ионов различного заряда от энергии налетающей альфа-частицы. Уменьшение при этом числа оставшихся электронов оболочки $5 p$ в результате процессов захвата электрона быстрой частицей приводит 
к существенному изменению соотношения сечений образования различного числа свободных электронов при ионизации Хе налетающими ионами $\mathrm{He}^{2+}$ в этих двух видах процессов по сравнению с процессом ионизации.

\section{Заключение}

Измерены абсолютные величины всех элементарных процессов, ведущих к образованию свободных электронов, при взаимодействии $\alpha$-частиц с атомами ксенона в диапазоне кинетических энергий налетающих ионов ${ }^{3} \mathrm{He}^{2+}$ от 1 до $100 \mathrm{keV}$. При столкновении иона c многоэлектронным атомом образование свободных электронов происходит в основном в многоэлектронных процессах, с высокой вероятностью сопровождающихся захватом электронов, т.е. в процессах захвата одного или двух электронов с дополнительной ионизацией многоэлектронного атома. Получены сечения образования свободных электронов в процессах ионизации, захвата одного и двух электронов. Определена область энергий столкновений, где меняется механизм образования свободных электронов. Показано, что сечения каналов процесса ионизации имеют близкую зависимость от энергии ионов $\mathrm{He}^{2+}$ и могут быть описаны универсальной зависимостью. Для процессов образования свободных электронов, обусловленных захватом электронов в процессе столкновения, подобные зависимости сечений каналов образования различного числа электронов от энергии наблюдаются только при энергиях столкновений, превышающих $\sim 55 \mathrm{keV}$. Это объясняется единым механизмом ионизации атомов мишени при высоких скоростях сближения сталкивающихся частиц.

\section{Финансирование работы}

Работа выполнена по Государственному заданию, тема № 0040-2019-0023.

\section{Конфликт интересов}

Авторы заявляют, что у них нет конфликта интересов.

\section{Список литературы}

[1] R.D. DuBois, S.T. Manson. Phys. Rev. A, 35, 2007 (1987).

[2] R.D. DuBois. Phys. Rev. A, 39, 4440 (1989).

[3] R.D. DuBois. Phys. Rev. A, 36, 2585 (1987).

[4] M.E. Rudd, T.V. Coffi, A. Itoh. Phys. Rev. A, 32, 2128 (1985).

[5] C.D. Archubi, C.C. Montanari, J.E. Miraglia. J. Phys. B: At. Mol. Opt. Phys., 40, 943 (2007).

[6] T. Kirchner, M. Horbasch, H.J. Ludde. Phys. Rev. A, 66, 052719 (2002)

[7] A. Amaya-Tapia, A. Antillón, C.D. Estrada. J. Phys. B: At. Mol. Opt. Phys., 51, 115201 (2018).

[8] G.M. Sigaud, M.M. Sant'Anna, H. Luna, A.C.F. Santos, C. McGrath, M.B. Shah, E.G. Cavalcanti, E.C. Montenegro. Phys. Rev. A, 69, 062718 (2004).
[9] G. Schenk, T. Kirchner. Phys. Rev. A, 91, 052712 (2015).

[10] Т. Киршнер, Х. Тавара, И.Ю. Толтихина, А.Д. Уланцев, В.П. Шевелько, Т. Штулькер. ЖТФ, 76 (9), 22 (2006). [T. Kirchner, H. Tawara, I.Yu. Tolstikhina, A.D. Ulantsev, V.P. Shevelko, Th. Stoehlker. Tech. Phys., 51 (9), 1127 (2006).]

[11] A. Salop, J. Eichler. J. Phys. B: At. Mol. Phys., 12, 257 (1979).

[12] E.G. Cavalcanti, G.M. Sigaud, E.C. Montenegro, M.M. Sant'Anna, H. Schmidt-Bocking. J. Phys. B: At. Mol. Opt. Phys., 35, 3937 (2002).

[13] L. Gulyás, P.D. Fainstein, A. Salin. J. Phys. B: At. Mol. Opt. Phys., 28, 245 (1995).

[14] M. Fiori, A.B. Rocha, C.E. Bielschowsky, G. Jalbert, C.R. Garibotti. J. Phys. B: At. Mol. Opt. Phys., 39, 1751 (2006).

[15] T. Kirchner, L. Gulyás, H.J. Ludde, A. Henne, E. Engel, R.M. Dreizer. Phys. Rev. Lett., 79, 1658 (1997).

[16] Е.А. Соловьев. УФН, 157 (3), 437 (1989).

[17] R.K. Janev, L.P. Presnyakov. Phys. Rep., 70 (1), (1981).

[18] G.N. Ogurtsov, S.Yu. Ovchinnikov, J.H. Macek, V.M. Mikoushkin. J. Phys. B: At. Mol. Opt. Phys., 46, 175203 (2013).

[19] В.В. Афросимов, А.А. Мамаев, М.Н. Панов, Н.В. Федоренко. ЖТФ, 39, 159 (1969).

[20] В.В. Афросимов, А.А. Басалаев, М.Н. Панов. ЖТФ, 64, 22 (1994). [V.V. Afrosimov, A.A. Basalaev, M.N. Panov. Tech. Phys., 39, 989 (1994).]

[21] В.В. Афросимов, А.А. Басалаев, Г.Н. Огурцов, М.Н. Панов. ЖТФ, 84 (5), 14 (2014). [V.V. Afrosimov, A.A. Basalaev, G.N. Ogurtsov, M.N. Panov. Tech. Phys., 59 (5), 642 (2014).]

[22] А.А. Басалаев, Г.Н. Огурцов, М.Н. Панов. ЖТФ, 88 (7), 977 (2019). [A.A. Basalaev, G.N. Ogurtsov, M.N. Panov. Tech. Phys., 63 (7), 947 (2018).] 